\title{
Role of amygdala and hippocampus in the neural circuit subserving conditioned defeat in Syrian hamsters
}

\author{
Chris M. Markham, ${ }^{1}$ Stacie L. Taylor, and Kim L. Huhman \\ Neuroscience Institute, Georgia State University, Atlanta, Georgia 30302, USA
}

\begin{abstract}
We examined the roles of the amygdala and hippocampus in the formation of emotionally relevant memories using an ethological model of conditioned fear termed conditioned defeat (CD). Temporary inactivation of the ventral, but not dorsal hippocampus ( $\mathrm{VH}, \mathrm{DH}$, respectively) using muscimol disrupted the acquisition of $\mathrm{CD}$, whereas pretraining $\mathrm{VH}$ infusions of anisomycin, a protein synthesis inhibitor, failed to block $C D$. To test for a functional connection between the VH and basolateral amygdala (BLA), we used a classic functional connectivity design wherein injections are made unilaterally in brain areas either on the same or opposite sides of the brain. A functional connection between the BLA and VH necessary for the acquisition of CD could not be found because unilateral inactivation of either BLA alone (but not either $\mathrm{VH}$ alone) was sufficient to disrupt $\mathrm{CD}$. This finding suggested instead that there may be a critical functional connection between the left and right BLA. In our final experiment, we infused muscimol unilaterally in the BLA and assessed Fos immunoreactivity on the contralateral side following exposure to social defeat. Inactivation of either BLA significantly reduced defeat-induced Fos immunoreactivity in the contralateral BLA. These experiments demonstrate for the first time that whereas the $\mathrm{VH}$ is necessary for the acquisition of $\mathrm{CD}$, it does not appear to mediate the plastic changes underlying $C D$. There also appears to be a critical interaction between the two BLAs such that bilateral activation of this brain area must occur in order to support fear learning in this model, a finding that is unprecedented to date.
\end{abstract}

Our laboratory has taken a novel approach to examine the behavioral and physiological changes that accompany social experience by studying a striking behavioral response that is exhibited following social defeat. When a Syrian hamster is paired with a larger, more aggressive opponent and is defeated, it subsequently becomes highly submissive and fails to defend its own home cage even against a smaller, nonaggressive intruder. We call this change in the behavior of the defeated hamster conditioned defeat (CD) (Portegal et al. 1993) and believe that it is a valuable model with which to study neural and behavioral plasticity following exposure to a biologically relevant stressor.

One of the critical structures subserving CD is the amygdala; temporary inactivation of its major subnuclei, including the basolateral amygdala (BLA), blocks the acquisition of CD (Jasnow and Huhman 2001). Together with the findings that protein synthesis inhibition in the BLA effectively disrupts CD (Markham and Huhman 2008) and that overexpression of cAMP response element binding protein (CREB) in the BLA enhances CD (Jasnow et al. 2005), the data support the hypothesis that the BLA is a critical site for plasticity related to CD.

One brain region that we have largely overlooked, but which receives considerable attention for its role in learning and memory, is the hippocampus. Several groups have now gathered anatomical and behavioral data demonstrating functionally specific dissociation between the dorsal $(\mathrm{DH})$ and ventral $(\mathrm{VH})$ regions of the hippocampus (Risold and Swanson 1996; Moser and Moser 1998; Bannerman et al. 2004; McEown and Treit 2009). While the DH is critical for spatial relationships (O'Keefe and Nadel 1978; Moser et al. 1993; Eichenbaum 1996) and has been shown to play an important role in social recognition in hamsters (Lai et al. 2005),

\footnotetext{
'Corresponding author.
}

E-mail psycmm@langate.gsu.edu; fax (404) 651-3929.

Article is online at http://www.learnmem.org/cgi/doi/10.1101//m.1633710. the $\mathrm{VH}$ appears to be involved in the production of behaviors produced in response to aversive stimuli (Trivedi and Coover 2004; Pentkowski et al. 2006).

Considering how critically important the hippocampus and amygdala are in relation to fear and memory, some studies are beginning to suggest that these areas may functionally interact to modulate memory function (Akirav and Richter-Levin 2002; McGaugh et al. 2002; McGaugh 2004; Vouimba et al. 2007). The BLA projects to the hippocampus (Amaral and Insausti 1992), and high-frequency stimulation of the BLA combined with tetanic stimulation of the perforant pathway facilitates hippocampal longterm potentiation (LTP) (Ikegaya et al. 1996). Additionally, electrolytic lesions of the $\mathrm{VH}$ produce a deficit in the acquisition of fear to a contextual conditioned stimulus, and NMDA lesions of the BLA cause a nonselective deficit in the acquisition of fear to both contextual and acoustic conditioned stimuli (Maren and Fanselow 1995). Although our laboratory has previously demonstrated that the BLA is critically involved in the acquisition of CD (Jasnow and Huhman 2001), the role of the hippocampus has yet to be investigated. The aim of the present study was to examine whether the $\mathrm{VH}$ and $\mathrm{DH}$ are involved in mediating $\mathrm{CD}$ and also to determine whether there is a functional interaction between the hippocampus and the amygdala in the acquisition of CD.

\section{Results}

\section{Histological analysis}

Figure 1A shows the cannula placements for animals infused with muscimol or vehicle into the DH (Experiment 1). Location of the needle tips was confined primarily to the dorsal regions of the DH. However, five animals were removed from the study due to misplaced or occluded cannula. Figure 1B shows the location of the cannula for animals receiving bilateral infusions into the $\mathrm{VH}$ (Experiments 2 and 3). Needle tips were confined to the CA1, CA2, 


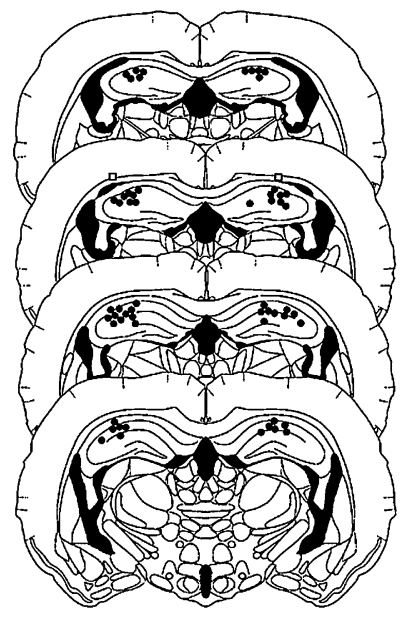

(A)

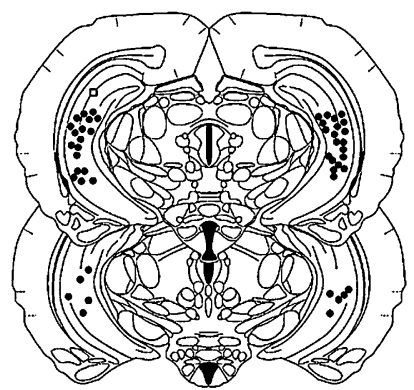

(B)
Figure 1. Histological recreation of injection sites of animals receiving infusions of muscimol into the $(A) \mathrm{DH}$ and $(B) \mathrm{VH}$. (Dots) The site of injection in one or more animals; (squares) misplaced injection sites. (Drawings are adapted from Morin and Wood [2001] and reprinted here with permission from Elsevier (C2001.)

and CA3 subregions of the $\mathrm{VH}$, while avoiding the dentate gyrus (DG) as well as the polymorph layer of the DG. Animals with misplaced or occluded cannula (nine in Experiment 2; two in Experiment 3) were removed from the study. Finally, Figure 2 shows the placements of the cannula aimed at the VH (Fig. 2A) and BLA (Fig. 2B; Experiments 4 and 5). Subjects with misplaced $(n=17 ; \mathrm{VH}=5 ; \mathrm{BLA}=12)$, damaged $(n=6)$, or occluded $(n=4)$ cannula were not included in the statistical analysis.

\section{Experiment 1: Muscimol infused into the DH does not reduce the acquisition of conditioned defeat} A total of 36 animals were used in the statistical analysis: vehicle $(n=9), 1.1 \mathrm{nmol}(n=7), 2.2 \mathrm{nmol}(n=12)$, and $3.3 \mathrm{nmol}(n=8)$. Infusion of muscimol into the $\mathrm{DH}$ immediately before defeat did not reduce the display of submissive behaviors $\left(F_{(3,35)}=\right.$ $0.072 ; P>0.05$ ) (Fig. 3). In addition, there were no significant differences observed in aggressive, social, and nonsocial behaviors (Fig. 3). Information about animals dropped from each experiment is given in the Histological analysis section. Additionally, no animals had to be removed from any experiment due to injury.

\section{Experiment 2: Muscimol infused into the $\mathrm{VH}$ reduces the acquisition of conditioned defeat}

A total of 32 animals were used in the statistical analysis: vehicle $(n=8), 1.1 \mathrm{nmol}(n=8), 2.2 \mathrm{nmol}(n=9)$, and $2.7 \mathrm{nmol}(n=7)$. Infusion of muscimol immediately before training significantly reduced the display of submissive behaviors during subsequent testing $\left(F_{(3,31)}=4.096 ; P<0.05\right)$ (Fig. 4$)$ without altering the behavior of either the resident aggressors or subjects during training. Post-hoc analysis showed that infusion of muscimol into the $\mathrm{VH}$ reduced submissiveness during testing at three doses when compared with animals injected with vehicle $(P<0.05$ for each comparison). Infusion of muscimol also significantly increased nonsocial behavior at all three doses, which consisted of normal locomotor activity and grooming $\left(F_{(3,31)}=7.895 ; P<0.05\right)$. Finally, there were no significant differences in aggressive and social behaviors, indicating that the inhibition of submissive behavior by muscimol was not due to a nonspecific decrease in agonistic or social behaviors.

\section{Experiment 3: Anisomycin infused in the $\mathrm{VH}$ does not reduce the acquisition of conditioned defeat}

A total of 30 animals were used in the statistical analysis: vehicle $(n=12)$ and anisomycin $(n=18)$. As shown in Figure 5 , infusion of anisomycin 30 min prior to defeat did not cause a significant reduction in submissive behaviors when animals were tested against a nonaggressive intruder (NAI) $24 \mathrm{~h}$ later $\left(F_{(1,29)}=0.395\right.$; $P>0.05)$. This dose of anisomycin was identical to that used in previous studies in which this concentration was effective in impairing contextual fear conditioning in the hippocampus (Rudy and Matus-Amat 2005) and acquisition of CD in the BLA (Markham and Huhman 2008).

\section{Experiment 4: Inactivation of the BLA-VH circuit inhibits the acquisition of conditioned defeat}

A total of 66 animals were used in the statistical analysis. Because there was no evidence of laterality (no significant differences between injections of the left and right hemispheres), groups were collapsed over side of injection and combined for subsequent analysis: Control Group: vehicle in contralateral BLA and $\mathrm{VH}(n=$ 11); Ipsilateral Group: muscimol in BLA and $\mathrm{VH}$ in the same hemisphere $(n=13)$; Contralateral Group: muscimol in contralateral BLA and VH $(n=12)$; Unilateral BLA Group: muscimol on one side of the BLA and vehicle in the contralateral VH $(n=14)$; and Unilateral VH Group: muscimol on one side of the $\mathrm{VH}$ and vehicle in the contralateral BLA $(n=16)$.

There was a significant effect of treatment on the display of submissive $\left(F_{(4,61)}=5.754 ; P<0.001\right)$ and social $\left(F_{(4,61)}=9.795 ; P<\right.$ 0.0001) behaviors during testing with the NAI $24 \mathrm{~h}$ later (Fig. 6). Treatment did not affect the initial defeat training experience as indicated by the fact that the durations of aggressive, social, and nonsocial behavior of the resident aggressor toward the experimental

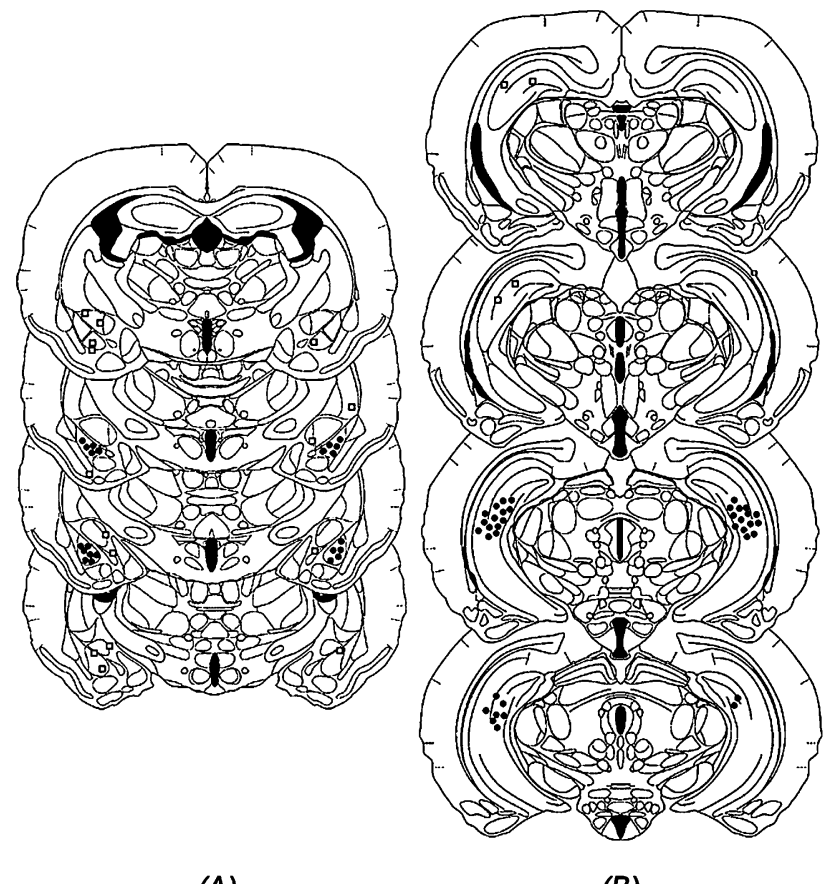

(A)

(B)

Figure 2. Histological recreation of injection sites of animals receiving infusion of muscimol into the (A) BLA and (B) VH. (Dots) The site of injection in one or more animals; (squares) misplaced injection sites. (Drawings are adapted from Morin and Wood [2001] and reprinted here with permission from Elsevier (02001.) 

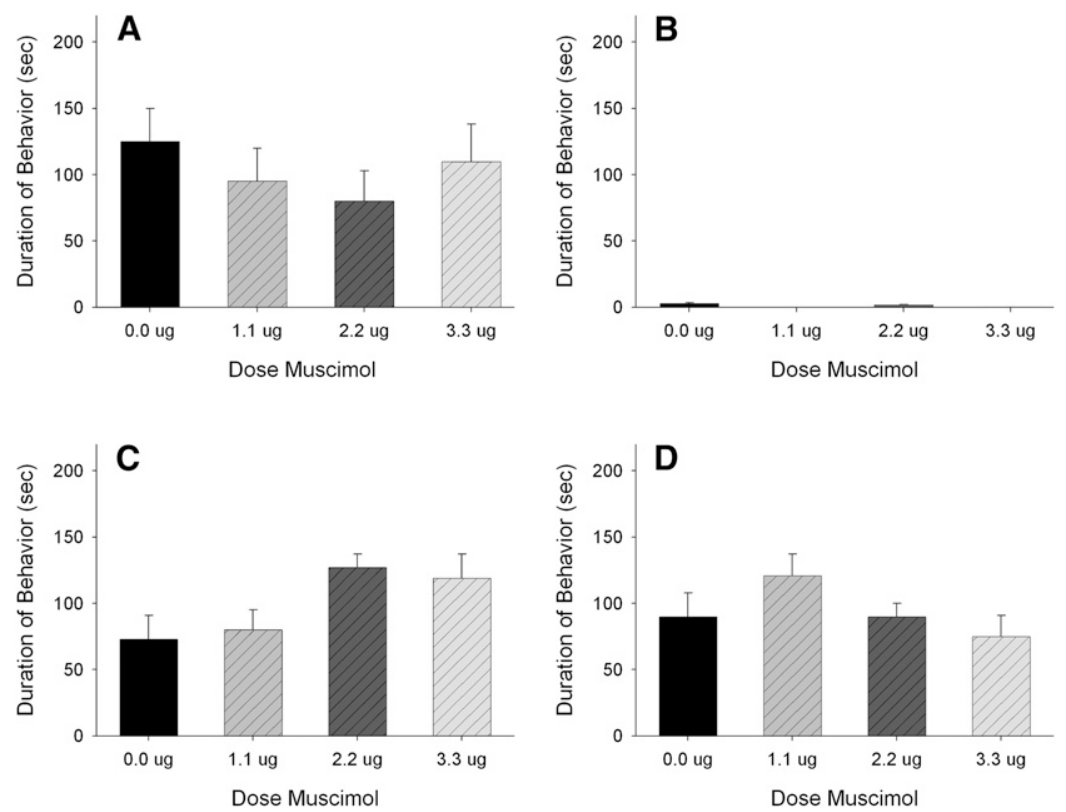

Figure 3. Total duration (mean \pm SEM) of submissive, aggressive, social, and nonsocial behavior displayed by defeated hamsters during a 5 -min test with a nonaggressive intruder. Animals received bilateral infusions of muscimol in the $\mathrm{DH}$ immediately before being defeated for $15 \mathrm{~min}$. There was no effect of drug on any behavior class.

animal were similar among the groups regardless of treatment condition (data not shown). A Tukey-Kramer multiple comparison post-hoc test indicated that unilateral inactivation of the BLA, regardless of group (or other area injected), significantly reduced the duration of submissive behaviors compared to vehicle and $\mathrm{VH}$ only inactivation groups $(P<0.01)$. No instances of aggression toward the NAI were observed, and there were no significant differences among groups in nonsocial behaviors.
VMH, LSv, and the Arcuate nucleus. Table 1 shows that aside from the BLA and LSv, no other structure showed significant differences in the number of Fos-positive cells when comparing the Saline/Defeat and Muscimol/Defeat groups.

\section{Discussion}

These experiments demonstrate that the ventral hippocampus is a component of the neural circuit subserving CD in that temporary inactivation of the ventral but not the dorsal hippocampus blocks the acquisition of conditioned defeat. Additionally, it first appeared that disrupting the VH-BLA pathway also significantly impairs the acquisition of CD; however, this attenuation appears to be due to the fact that unilaterally inactivating the BLA alone (originally performed as a control group) is sufficient to inhibit the acquisition of $\mathrm{CD}$. This finding suggests the possibility that there is functional connectivity between the left and right BLA such that neural activity in both nuclei must occur in order to mediate the behavioral plasticity associated with a defeat experience. We began to examine this possibility in our final experiment wherein we demonstrated that unilateral inactivation of the BLA suppresses Fos-immunoreactivity (Fos-IR) in the contralateral BLA following social defeat. Our findings thus provide strong evidence to support the hypothesis that there is a functional connection between the left and right BLA such that inactivating one side of the BLA effectively inhibits the other.

The data from a recent study by Lai and colleagues (2005) showing that temporary inactivation of the $\mathrm{DH}$ attenuates the

\section{Experiment 5: Unilateral} inactivation of BLA suppresses Fos activity in contralateral BLA

A total of 16 animals were used in the statistical analysis: Vehicle Defeat $(n=5)$, Muscimol Defeat $(n=6)$, and Muscimol No Defeat $(n=5)$. Because there were no significant differences in Fos immunoreactivity (Fos-IR) based on the side of muscimol injection (left or right BLA), these groups were combined. As shown in Figure 7, there was a significant effect of treatment on Fos-IR in the contralateral $\operatorname{BLA}\left(F_{(2,13)}=24.66 ; P<0.0001\right)$. Post-hoc tests showed that both the Muscimol/No Defeat and Muscimol Defeat groups had significantly lower levels of Fos-positive cells compared to the Vehicle/Defeat animals in the BLA opposite the injection site. No significant differences were found between the Muscimol/No Defeat and Muscimol/Defeat groups.

In order to ensure that muscimol infusions alone did not cause a global or nonspecific inhibition of neural activity, Fos-positive cells were also counted in brain areas in close proximity to the BLA, including the CeA, MeA, BNST,
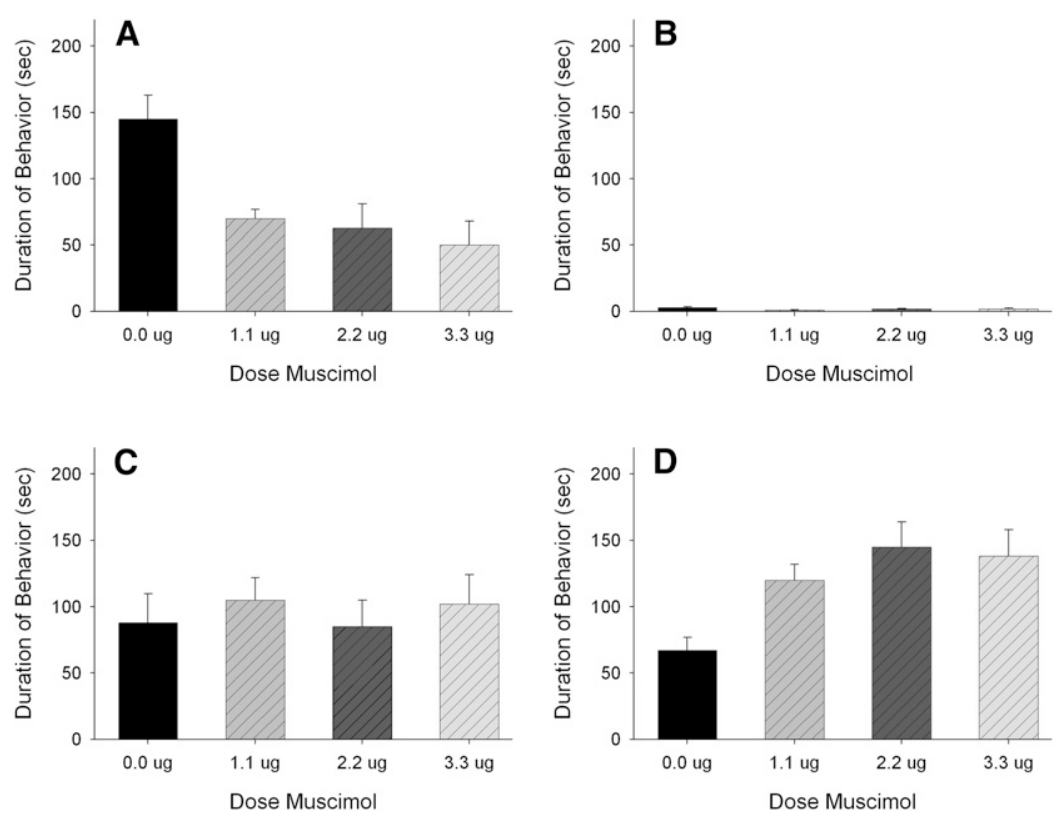

Figure 4. Total duration (mean $\pm \mathrm{SEM}$ ) of submissive, aggressive, social, and nonsocial behavior displayed by defeated hamsters during a 5 -min test with a nonaggressive intruder. Animals received bilateral infusions of muscimol in the VH immediately before being defeated for 15 min. Significant differences are indicated by unshared letters $(P<0.05)$. 


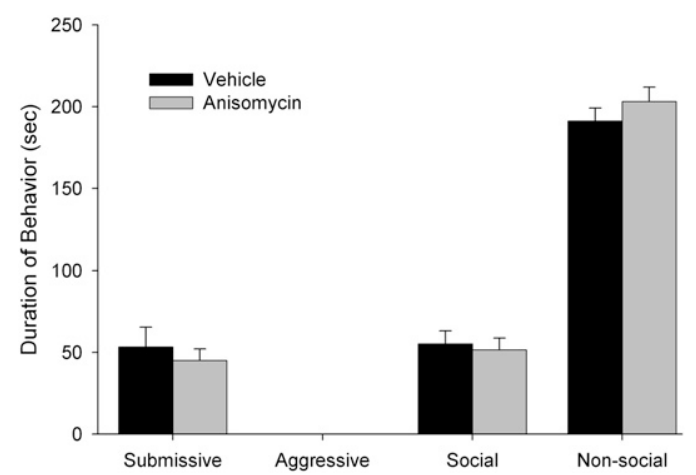

Figure 5. Total duration (mean \pm SEM) of submissive, aggressive, social, and nonsocial behavior displayed by defeated hamsters during a 5-min test with a nonaggressive intruder. Animals received bilateral infusions of anisomycin in the $\mathrm{VH}$ immediately before being defeated for $15 \mathrm{~min}$. There was no effect of drug on any behavior class.

avoidance by defeated hamsters of an animal that had previously defeated them led us to hypothesize that the DH would also be involved in CD. Instead, the results of Experiment 1 showed that muscimol infusion in the $\mathrm{DH}$ fails to inhibit the acquisition of $\mathrm{CD}$, suggesting that the $\mathrm{DH}$ is not a critical component of the neural circuit mediating conditioned defeat. In the Lai et al. (2005) study, hamsters were exposed to the familiar, dominant opponent. In contrast, in our studies the stimulus animal (the nonaggressive intruder) is very different in size, age, and behavior from the resident aggressor used during the initial defeat training. Thus, it appears that the DH may be involved in behavioral responses to a particular dominant opponent, but it does not appear to be involved in the acquisition of generalized behavioral changes that are produced in response to a defeat experience, per se. The differences between the two models may therefore explain why inactivation of the $\mathrm{DH}$ failed to inhibit the acquisition of $\mathrm{CD}$. We do agree that the $\mathrm{DH}$ is an integral component of fear conditioning circuitry subserving some of the more traditional models, particularly wherein specific context cues are relevant. Another possible explanation for our failure to observe a role for the $\mathrm{DH}$ is that muscimol infusions may have caused an increase in general activity levels during defeat and that this increase interfered with the normal pattern of defeat. Indeed, damage to the hippocampus has been shown in some studies (Yoon and Otto 2007) to increase locomotor activity. Careful analysis of the defeat sessions, however, revealed no such locomotor changes.

Our finding that infusion of muscimol into the $\mathrm{VH}$ inhibits the acquisition of $\mathrm{CD}$ is consistent with several other studies showing that this area is important in the production of anxiety-like behaviors. For example, electrolytic or neurotoxic lesions of the $\mathrm{VH}$ have been shown to have anxiolytic effects, including a reduction in the avoidance of the open arms of an elevated T-maze (Trivedi and Coover 2004), reduced avoidance of a male conspecific in the social interaction test (Deacon et al. 2002), reduced anxiety-like behaviors in a social interaction test (McHugh et al. 2004), and an impairment of risk assessment behaviors (a behavioral index of anxiety) in rats confronted with an ethologically relevant threat stimulus (Pentkowski et al. 2006). In addition to its role in regulating anxiety-like behaviors, a number of recent studies have demonstrated that the VH may also be critically involved in certain forms of conditioned fear (Rogers et al. 2006; Yoon and Otto 2007; Czerniawski et al. 2009). The study by Czerniawski et al. (2009) demonstrated a double-dissociation between the $\mathrm{DH}$ and $\mathrm{VH}$ as temporary inactivation of the ventral, but not dorsal, hippocampus blocked the acquisition and expression of trace fear conditioning, whereas inactivation of the dorsal, but not ventral, hippocampus impaired the behavioral response to a delayed reinforced alternation task.

Although the previously mentioned findings, combined with our own work, provide strong evidence for the involvement of the $\mathrm{VH}$ in conditioned fear responses, few if any studies have specifically examined whether actual plasticity and memory consolidation occurs in the $\mathrm{VH}$. Although there has been some recent controversy concerning the use of protein synthesis inhibitors to disrupt learning (Gold 2008), this technique is still widely used and accepted. We have previously demonstrated that infusion of anisomycin into the BLA blocks memory formation necessary for CD (Markham and Huhman 2008). Infusion of this same dose of anisomycin into the $\mathrm{VH}$ in the present study was ineffective. The fact that we found anisomycin to be effective in the BLA but not the $\mathrm{VH}$ argues against a nonspecific effect of anisomycin on the well-being and function of neurons explaining the effectiveness of the treatment in blocking memory formation for CD. Together, our data suggest that while the $\mathrm{VH}$ is an integral component of the neural circuit mediating the acquisition of $\mathrm{CD}$, actual plasticity related to the defeat experience likely occurs elsewhere (e.g., the BLA). Indeed, the VH has reciprocal connections to multiple brain regions that are critical in fear, anxiety, and stress responsivity (Moser and Moser 1998; Bast et al. 2001; Petrovich et al. 2001) such as the amygdala. We must also acknowledge, however, the possibility that the VH is also a critical site for plasticity underlying $\mathrm{CD}$ but that this plasticity is not dependent on protein synthesis.

We were initially surprised at the finding in Experiment 4 that temporary inactivation of the VH-BLA circuit in both the ipsilateral
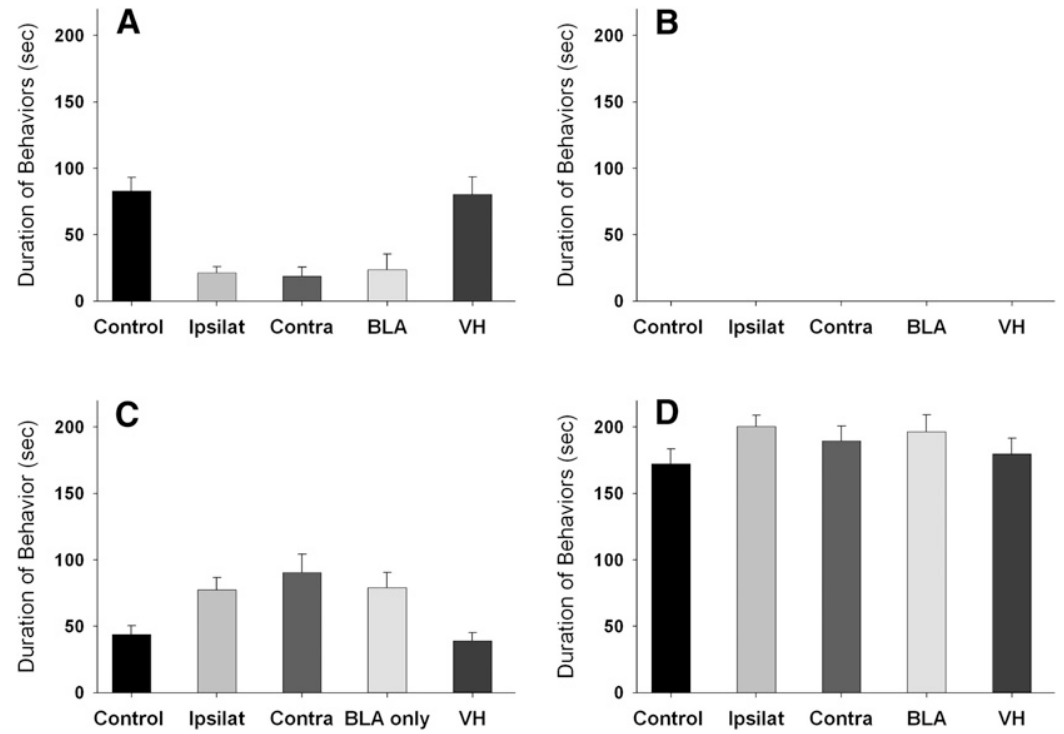

Figure 6. Total duration (mean \pm SEM) of submissive, aggressive, social, and nonsocial behavior displayed by defeated hamsters during a 5-min test with a nonaggressive intruder. Animals received infusions of muscimol in the VH and/or BLA immediately before being defeated for $15 \mathrm{~min}$. Significant differences are indicated by unshared letters $(P<0.05)$. 



Figure 7. Photomicrographs of Fos-immunoreactive cells (dark dots) in the basolateral amygdala (BLA) and surrounding areas in the (A) saline/defeat, (B) muscimol/defeat, and (C) muscimol/no defeat groups.

and contralateral groups caused a significant reduction in the acquisition of $\mathrm{CD}$. This suggested to us that the projections between these structures may, in fact, be both ipsilateral and contralateral instead of mainly ipsilateral, which had been suggested in the literature (Packard et al. 1994). In fact, it appears that this surprising observation can actually be explained by the equally unexpected finding that unilateral inactivation of the BLA (but not VH) significantly inhibits the acquisition of $\mathrm{CD}$. Several key conclusions can be based on these results. First, these findings indicate that, at least with the conditioned defeat model, the left and right $\mathrm{VH}$ can function independently to support learning while the left and right BLA cannot. Second, there does not appear to be any laterality of function within the hamster amygdala, or, alternatively, the left and right BLA are connected such that any laterality of function is masked. Indeed, there is some evidence for this connection, and it has been suggested that it occurs via the anterior commissure, at least in rats (Martinez-Lorenzana et al. 2004). Our final experiment sought to indirectly test the existence of this connection by unilaterally inactivating one BLA and examining Fos-IR in the contralateral BLA. As reported above, this inactivation caused a suppression of Fos opposite to the side of the infusion, suggesting that unilateral inactivation of the BLA is sufficient to disrupt $\mathrm{CD}$ because it also inhibits activation of the contralateral BLA. Importantly, this suppression was specific to the BLA, as Fos counts in the neighboring regions in muscimolinfused animals (with the exception of the ventral lateral septum; LSv) were not significantly different from the counts obtained from vehicle-infused hamsters. These findings seem to contradict previous reports showing that conditioned fear can be supported even after unilateral lesions of the amygdala (LaBar and LeDoux 1996; Goosens and Maren 2001). There are other examples, however, where bilateral activation of a brain region is required for the expression of a particular behavior. For example, Ferris and colleagues (1994) demonstrated that bilateral activity of the anterior hypothalamus $(\mathrm{AH})$ is critical for the expression of flankmarking behavior in hamsters.

In conclusion, the results of the present series of experiments, combined with previous findings from our laboratory (Jasnow and Huhman 2001; Huhman et al. 2003; Jasnow et al. 2005; Markham and Huhman 2008), indicate that while several neural sites are important mediators of CD, the BLA appears, thus far, to be the key site wherein plasticity related to the defeat experience occurs. Additionally, we provide strong indirect evidence that the left and right BLA form a functional unit that is necessary for the mediation of $\mathrm{CD}$.

Table 1. Counts of Fos-positive cells

\begin{tabular}{lcccc}
\hline Region & $\begin{array}{c}\text { Bregma } \\
(\mathbf{m m})\end{array}$ & $\begin{array}{c}\text { Saline/Defeat } \\
(\boldsymbol{n}=5)\end{array}$ & $\begin{array}{c}\text { Muscimol/Defeat } \\
(\boldsymbol{n}=\mathbf{6})\end{array}$ & $\begin{array}{c}\text { Muscimol/No } \\
\text { Defeat }(\boldsymbol{n}=\mathbf{5})\end{array}$ \\
\hline BLA & -1.50 & $167 \pm 24$ & $75 \pm 9^{\mathrm{a}, \mathrm{c}}$ & $28 \pm 9^{\mathrm{a}}$ \\
CeA & -1.20 & $28 \pm 7$ & $23 \pm 6$ & $2 \pm 0.9^{\mathrm{a}, \mathrm{b}}$ \\
MeApv & -1.50 & $245 \pm 60$ & $193 \pm 16$ & $80 \pm 12^{\mathrm{a}, \mathrm{b}}$ \\
MeApd & -1.50 & $130 \pm 43$ & $93 \pm 8$ & $28 \pm 5^{\mathrm{a}, \mathrm{b}}$ \\
BNSTpm & +0.5 & $83 \pm 12$ & $67 \pm 14$ & $26 \pm 5^{\mathrm{a}, \mathrm{b}}$ \\
BNSTpi & +0.5 & $109 \pm 22$ & $96 \pm 24$ & $28 \pm 3^{\mathrm{a}, \mathrm{b}}$ \\
LSV & +0.9 & $396 \pm 60$ & $290 \pm 55$ & $108 \pm 41^{\mathrm{a}}$ \\
VMHdm & -2.0 & $83 \pm 17$ & $67 \pm 7$ & $24 \pm 4^{\mathrm{a}, \mathrm{b}}$ \\
Arc & -2.0 & $116 \pm 43$ & $103 \pm 10$ & $40 \pm 8^{\mathrm{a}, \mathrm{b}}$ \\
\hline
\end{tabular}

The region of interest was counted using the adjoining Nissl-stained section.

${ }^{a} P<0.05$ versus saline/defeat animals.

${ }^{\mathrm{b}} P<0.01$ versus muscimol/defeat animals.

${ }^{c} P<0.01$ versus muscimol/no defeat animals.

Summary of Fos immunoreactivity in select brain regions. Data show the mean number of c-Fos immunoreactive cells located in representative sections of each brain region (SEM). The region of interest was counted using adjoining the Nissl-stained sections at $20 \times$ magnification. Significant differences are indicated by unshared letters. 


\section{Materials and Methods}

\section{Animals and housing conditions}

Male Syrian hamsters (Mesocricetus auratus) weighing 120-140 g at the beginning of the experiment were obtained from Charles River Laboratories (Kingston, NY). Older males ( $>6 \mathrm{mo}$ ) weighing 160-180 g were housed individually and used as resident aggressors during the defeat training. Younger males $(2 \mathrm{mo})$ that weighed 100-110 g were group housed (five to six hamsters per cage) and used as nonaggressive intruders during CD testing. All hamsters were housed in polycarbonate cages $(20 \times 40 \times 20 \mathrm{~cm})$ with wire mesh tops, and food and water were available ad libitum. Animals were kept in a temperature- and humidity-controlled room with a 14:10-h light:dark cycle (lights out at $1100 \mathrm{~h}$ ). All procedures and protocols were approved by the Georgia State University Institutional Animal Care and Use Committee, and all methods were in accordance with the standards outlined in the National Institutes of Health Guide for Care and Use of Laboratory Animals.

\section{Surgical procedures}

Hamsters were deeply anesthetized with sodium pentobarbital $(90 \mathrm{mg} / \mathrm{kg}$, i.p.) and placed into a stereotaxic frame. All animals were implanted with $4-\mathrm{mm}, 26$-gauge stainless steel cannula (Plastics One). Lambda and bregma were leveled prior to placement of the guide cannula. Guide cannula were bilaterally implanted (with the exception of Experiments 4 and 5) and aimed at the DH (Experiment 1), VH (Experiments 2 and 3), the BLA and VH (Experiment 4), or the BLA (Experiment 5). Following surgery, dummy stylets were placed in the guide cannula to help maintain patency. Hamsters were allowed 7-10 d to recover from surgery prior to the start of behavioral testing. They were handled each day after surgery by gently restraining them and removing and replacing the dummy stylet in order to habituate the subjects to the injection procedure.

\section{Drug infusion}

Infusions into the $\mathrm{DH}, \mathrm{VH}$, and BLA were administered to freely moving hamsters over 2 min with a Hamilton syringe mounted on a syringe pump (Harvard Apparatus) connected to a 33-gauge injection needle via polyethylene tubing (Fisher Scientific). The needle was kept in place for an additional $1 \mathrm{~min}$ before being removed to ensure complete diffusion of the drug after which the dummy stylet was replaced. Hamsters were infused with either the $\mathrm{GABA}_{\mathrm{A}}$ agonist muscimol (Sigma; Experiments 1, 2, 4, and 5), the protein synthesis inhibitor anisomycin (Sigma; Experiment 3), or vehicle (physiological saline; used as a control in all experiments).

\section{Social defeat and testing}

Subjects were tested in the conditioned defeat model, described in detail elsewhere (Huhman et al. 2003). All training and testing sessions were performed during the first $3 \mathrm{~h}$ of the dark phase of the light-dark cycle to minimize circadian variation in the behavior and physiology. Training consisted of one 15-min exposure to the resident aggressor (RA) in the aggressor's home cage, upon which the RA reliably attacked the experimental hamsters within $60 \mathrm{sec}$. We employ a caveat wherein any hamster that is bitten such that it bleeds is removed from the study and is examined by a veterinarian, but none of the subjects were excluded for this reason. The following day, animals were paired in their own home cage for $5 \mathrm{~min}$ with a nonaggressive intruder (NAI). An animal was considered to show conditioned defeat if it exhibited an increase in submissive and defensive behaviors and no aggressive behaviors when the NAI was introduced into its home cage.

\section{Behavioral analysis}

All training and testing sessions were videotaped via a camera mounted overhead. The video files were scored by an experimentally blind observer using the behavioral scoring program Hindsight (developed by Dr. Scott Weiss). The total duration of four classes of behavior were scored during the test session: (1) social behavior (stretch, approach, sniff, nose touching, and flank marking); (2) nonsocial behavior (locomotion, exploration, grooming, nesting, feeding, and sleeping); (3) submissive/defensive behaviors (flight, avoidance, tail up, upright, side defense, full submissive posture, stretch attend, head flag, attempted escape from cage); and (4) aggressive behaviors (upright and side offense, chase and attack, including bite). For all experiments, the behavior of the resident aggressors and subjects was also scored during training to ensure that (1) the presence of a drugged subject during training did not alter the behavior of the resident aggressor; (2) all animals received similar defeats; and (3) subjects were able to produce normal submissive behavior in response to attack.

\section{Site verification}

At the end of Experiments 1-4, hamsters were administered an overdose of sodium pentobarbital and infused with either $200 \mathrm{~nL}$ (BLA) or $300 \mathrm{~nL}$ (DH and VH) of India ink to verify the placement of the needle. The brains were removed and post-fixed in $10 \%$ buffered formalin for $3 \mathrm{~d}$ before being sectioned on a cryostat. Thirty-micrometer sections were taken and stained with neutral red and coverslipped with DPX (VWR International Ltd.). Sections were then examined under a light microscope for placement verification. Only animals with injection sites within $0.3 \mathrm{~mm}$ of the target structure were included in the statistical analysis.

\section{Immunohistochemistry}

In Experiment 5, animals were administered an overdose of sodium pentobarbital $60 \mathrm{~min}$ after the end of defeat and were infused with $200 \mathrm{~nL}$ of India ink to verify the placement of the needle. Subjects were then perfused transcardially with $0.1 \mathrm{M}$ phosphate-buffered saline (PBS) and $4 \%$ paraformaldehyde. Brains were post-fixed in $4 \%$ paraformaldehyde overnight and switched to a $30 \%$ sucrose-paraformaldehyde solution and kept at $4{ }^{\circ} \mathrm{C}$ for an additional $48 \mathrm{~h}$. Brains were blocked on the coronal plane and sectioned on a cryostat to a thickness of $30 \mu \mathrm{m}$. Consecutive sections were placed sequentially across four vials filled with cyroprotectant. The sections covered the areas between the anterior bed nucleus of the stria terminalis (BNST) and the posterior division of the BLA. Sections were labeled for c-fos using primary antisera directed against the protein product of the immediate early gene c-fos (rabbit anti-c-fos polyclonal antibody, 1:20000; Santa Cruz Biotechnology).

All washes, rinses, and incubations were performed in 12well tissue culture plates, which were gently shaken on an orbital shaker throughout the immunostaining procedure. Briefly, sections were rinsed in $0.1 \mathrm{M}$ PBS and incubated in a $1 \%$ hydrogen peroxide solution for $15 \mathrm{~min}$, followed by a PBS wash. Sections were then incubated for $1 \mathrm{~h}$ in the primary antibody at room temperature, and then for $48 \mathrm{~h}$ at $4^{\circ} \mathrm{C}$. Following incubation with the primary antibody, the sections were rinsed in PBS and incubated for $1 \mathrm{~h}$ in a $0.4 \%$ solution of Triton X-100 in $0.1 \mathrm{M}$ PBS containing the secondary antibody (biotintylated anti-rabbit IgG polyclonal antibody, 1:600; Vector Laboratories). They were then rinsed with PBS, followed by incubation for $1 \mathrm{~h}$ with an avidinbiotin complex reagent (Vectastain Elite ABC kit; Vector Laboratories). After rinsing with PBS and sodium acetate solution, sections were incubated in a nickel-3,3'-diaminobenzidene (DAB) solution for $10 \mathrm{~min}$ in order to produce a blue-black-stained product and then washed again with sodium acetate and PBS in order to halt the DAB reaction. Finally, the sections were mounted on gelatin-coated slides, dehydrated, and coverslipped with DPX (VWR International).

\section{Cell counting}

An observer blind to the treatment conditions performed all cell counts using brightfield microscopy at 10-20× magnification. For the quantification of Fos labeling, areas of interest were first delineated using adjoining Cresyl violet-stained sections. Foslabeled cells were counted within this standardized template for each brain region. The following areas were counted based on the hamster atlas of Morin and Wood (2001): BLA, medial amygdala (MeA), central amygdala (CeA), ventral lateral septum (LSv), the posteromedial and posterointermediate bed nucleus of the stria 
terminalis (BNSTpm and BNSTpi, respectively), ventromedial hypothalamus (VMH), and the arcuate nucleus (Arc).

\section{Experiments 1 and 2: DH/VH acquisition of conditioned defeat}

The purpose of Experiments 1 and 2 was to determine whether infusion of muscimol in the $\mathrm{DH}$ or $\mathrm{VH}$, respectively, would reduce the acquisition of CD. Animals (Experiment 1: $n=41$; Experiment 2: $n=41$ ) were randomly assigned to groups. Hamsters received either muscimol $(1.1,2.2,2.7$, or $3.3 \mathrm{nmol}$ in $300 \mathrm{~nL}$ of saline) or vehicle (physiological saline) into the $\mathrm{DH}$ or $\mathrm{VH}$ immediately before being placed in the cage of a resident aggressor for $15 \mathrm{~min}$. This range of dosages of muscimol is effective in blocking CD when given in the amygdala (Markham and Huhman 2008). On the following day, animals were tested for $5 \mathrm{~min}$ in their own home cage in the presence of a nonaggressive intruder, as described above.

\section{Experiment 3: VH/protein synthesis inhibition}

The aim of Experiment 3 was to determine whether the inhibition of protein synthesis via infusion of anisomycin in the $\mathrm{VH}$ before training would block the acquisition of CD. Animals $(n=32)$ were again randomly assigned to groups after being weight matched. Anisomycin $(125 \mu \mathrm{g} / \mu \mathrm{L})$ or vehicle was infused in hamsters 30 min prior to initial defeat and tested with a resident aggressor for $15 \mathrm{~min}$. This dose of anisomycin given into the BLA blocks the acquisition of CD in hamsters (Markham and Huhman 2008). On the following day, animals were tested for $5 \mathrm{~min}$ in their own home cage in the presence of a nonaggressive intruder.

\section{Experiment 4: VH/BLA acquisition of conditioned defeat}

The purpose of Experiment 4 was to determine whether there is a functional interaction between the BLA and VH in the acquisition of CD. Animals $(n=93)$ were randomly assigned to groups and infused with muscimol $(2.2 \mathrm{nmol}$ in $300 \mathrm{~nL}$ of saline in each injection site) or vehicle (physiological saline) 5 min before being placed in the cage of a resident aggressor for $15 \mathrm{~min}$. On the following day animals were tested for $5 \mathrm{~min}$ in their own home cage in the presence of a nonaggressive intruder. The animals were divided into five major groups that were then balanced for side of the injection: Control (contralateral saline infusions in either the right/left BLA and the left/right VH), Ipsilateral (muscimol infusions in either the left BLA and left $\mathrm{VH}$ or right BLA and right $\mathrm{VH}$ ), Contralateral (muscimol infusions in either the left BLA and right $\mathrm{VH}$ or right BLA and left $\mathrm{VH}$ ), Unilateral BLA (musimol infusion in either the right or left BLA and saline in the right or left $\mathrm{VH}$ ), and Unilateral $\mathrm{VH}$ (muscimol infusion on either the right or left $\mathrm{VH}$ and saline in the right or left BLA).

\section{Experiment 5: Unilateral muscimol BLA/ Fos expression}

The purpose of Experiment 5 was to further examine the novel possibility that there is a functional connection between the right and left BLA that is critical for the formation of CD. Animals $(n=$ 18) were randomly assigned to groups and unilaterally infused with muscimol ( $2.2 \mathrm{nmol}$ in $200 \mathrm{~nL}$ of saline) or vehicle in the left or right BLA. Fifteen minutes after the injection, subjects were placed into the home cage of a resident aggressor and were defeated for $15 \mathrm{~min}$ (defeat group) or left undisturbed in their own home cages for the same amount of time (undefeated group). All animals were then left undisturbed for $1 \mathrm{~h}$ after which they were injected with an overdose of sodium pentobarbital (90 mg/kg i.p.) and transcardially perfused. Brains were removed and stored in $10 \%$ paraformaldehyde for $48 \mathrm{~h}$ after which they were cut into $30-\mu \mathrm{m}$ sections on a cryostat.

\section{Statistical analysis}

The total duration (seconds) of each behavior displayed (Submissive/Defensive, Social, Nonsocial) was determined, and the mean total duration of each behavior was then compared using analysis of variance (ANOVA) with dose as the between-subjects factor. Statistically significant differences were further analyzed using a Tukey-Kramer multiple comparison post-hoc test to compare all pairwise differences among group means. The immunohistochemistry data were analyzed using two-way between-subjects ANOVAs, with drug injection and stimulus exposure as independent factors. Significant differences for all analyses were set at $P<0.05$.

\section{Acknowledgments}

We thank Alisa Norvelle and Daniel Erwin for their expert technical assistance. We also thank Raquel Martinez for her assistance in creating the photomicrograph of Fos-immunoreactive cells. This research was supported by the National Institutes of Health grant MH62044 to K.L.H., and in part by the Center for Behavioral Neuroscience, a National Science Foundation Science and Technology Center program under agreement no. IBN-9876754. All procedures were approved by the Georgia State University Animal Care and Use Committee and comply with U.S. law.

\section{References}

Akirav I, Richter-Levin G. 2002. Mechanisms of amygdala modulation of hippocampal plasticity. J Neurosci 22: 9912-9921.

Amaral DG, Insausti R. 1992. Retrograde transport of D- $\left[{ }^{3} \mathrm{H}\right]$-aspartate injected into the monkey amygdaloid complex. Exp Brain Res 88: 375 388 .

Bannerman DM, Rawlins JNP, McHugh SB, Deacon RMJ, Yee BK, Bast T, Zhang W-N, Pothuizen HHJ, Feldon J. 2004. Regional dissociations within the hippocampus-memory and anxiety. Neurosci Biobehav Rev 28: $273-283$.

Bast T, Zhang WN, Feldon J. 2001. The ventral hippocampus and fear conditioning in rats. Different anterograde amnesias of fear after tetrodotoxin inactivation and infusion of the $\mathrm{GABA}_{\mathrm{A}}$ agonist muscimol. Exp Brain Res 139: 39-52.

Czerniawski J, Yoon T, Otto T. 2009. Dissociating space and trace in dorsal and ventral hippocampus. Hippocampus 19: 20-32.

Deacon RM, Bannerman DM, Rawlins JN. 2002. Anxiolytic effects of cytotoxic hippocampal lesions in rats. Behav Neurosci 116: 494-497.

Eichenbaum H. 1996. Learning from LTP: A comment on recent attempts to identify cellular and molecular mechanisms of memory. Learn Mem 3: 61-73.

Ferris CF, Delville Y, Irvin RW, Portegal M. 1994. Septo-hypothalamic organization of a stereotyped behavior controlled by vasopressin in golden hamsters. Physiol Behav 55: 755-759.

Gold PE. 2008. Protein synthesis inhibition and memory: Formation vs. amnesia. Neurobiol Learn Mem 89: 201-211.

Goosens KA, Maren S. 2001. Contextual and auditory fear conditioning are mediated by the lateral, basal, and central amygdaloid nuclei in rats. Learn Mem 8: 148-155.

Huhman KL, Solomon MB, Janicki M, Harmon AC, Lin SM, Israel JE, Jasnow AM. 2003. Conditioned defeat in male and female Syrian hamsters. Horm Behav 44: 293-299.

Ikegaya Y, Saito H, Abe K. 1996. The basomedial and basolateral amygdaloid nuclei contribute to the induction of long-term potentiation in the dentate gyrus in vivo. Eur J Neurosci 8: 1833-1839.

Jasnow AM, Huhman KL. 2001. Activation of $\mathrm{GABA}_{\mathrm{A}}$ receptors in the amygdala blocks the acquisition and expression of conditioned defeat in Syrian hamsters. Brain Res 920: 142-150.

Jasnow AM, Shi C, Israel JE, Davis M, Huhman KL. 2005. Memory of social defeat is facilitated by cAMP response element-binding protein overexpression in the amygdala. Behav Neurosci 119: 1125-1130.

LaBar KS, LeDoux JE. 1996. Partial disruption of fear conditioning in rats with unilateral amygdala damage: Correspondence with unilateral temporal lobectomy in humans. Behav Neurosci 110: 991-997.

Lai WS, Ramiro LL, Yu HA, Johnston RE. 2005. Recognition of familiar individuals in golden hamsters: A new method and functional neuroanatomy. J Neurosci 25: 11239-11247.

Maren S, Fanselow MS. 1995. Synaptic plasticity in the basolateral amygdala induced by hippocampal formation stimulation in vivo. J Neurosci 15: 7548-7564.

Markham CM, Huhman KL. 2008. Is the medial amygdala part of the neural circuit modulating conditioned defeat in Syrian hamsters? Learn Mem 15: 6-12.

Martinez-Lorenzana G, Jimenez JR, Condes-Lara M. 2004. Interamygdaloid connection of basolateral nucleus through the anterior commissure in the rat. Neurosci Lett 366: 154-157.

McEown K, Treit D. 2009. The role of the dorsal and ventral hippocampus in fear and memory of a shock-probe experience. Brain Res 1251: 185-194.

McGaugh JL. 2004. The amygdala modulates the consolidation of memories of emotionally arousing experiences. Annu Rev Neurosci 27: 1-28. 
McGaugh JL, McIntyre CK, Power AE. 2002. Amygdala modulation of memory consolidation: Interaction with other brain regions. Neurobio Learn Mem 78: 539-552.

McHugh SB, Deacon RM, Rawlins JN, Bannerman DM. 2004. Amygdala and ventral hippocampus contribute differentially to mechanisms of fear and anxiety. Behav Neurosci 118: 63-78.

Morin LP, Wood RI. 2001. A stereotaxic atlas of the Golden hamster brain. Academic Press, San Diego, CA.

Moser MB, Moser E. 1998. Functional differentiation in the hippocampus. Hippocampus 8: 608-619.

Moser E, Moser MB, Andersen P. 1993. Spatial learning impairment parallels the magnitude of dorsal hippocampus lesions, but is hardly present following ventral lesions. J Neurosci 13: 3916-3925.

O'Keefe J, Nadel L. 1978. The hippocampus as a cognitive map. Oxford University Press, Oxford, UK.

Packard MG, Cahill L, McGaugh JL. 1994. Amygdala modulation of hippocampal-dependent and caudate nucleus-dependent memory processes. Proc Natl Acad Sci 91: 8477-8481.

Pentkowski NS, Blanchard DC, Lever C, Litvin Y, Blanchard RJ. 2006. Effects of lesions to the dorsal and ventral hippocampus on defensive behaviors in rats. Eur I Neurosci 21: 2185-2196.

Petrovich GD, Canteras NS, Swanson LW. 2001. Combinatorial amygdalar inputs to hippocampal domains and hypothalamic behavior systems. Brain Res Brain Res Rev 38: 247-289.
Portegal M, Huhman KL, Meyerhoff JL. 1993. Conditioned defeat in the Syrian hamster. Behav Neural Biol 60: 93-102.

Risold PY, Swanson LW. 1996. Structural evidence for functional domains in the rat hippocampus. Science 272: 1484-1486.

Rogers JL, Hunsaker MR, Kesner RP. 2006. Effects of ventral and dorsal CA1 subregional lesions on trace fear conditioning. Neurobiol Learn Mem 86: 72-81.

Rudy JW, Matus-Amat P. 2005. The ventral hippocampus supports a memory representation of context and contextual fear conditioning: Implications for a unitary function of the hippocampus. Behav Neurosci 119: 154-163.

Trivedi MA, Coover GD. 2004. Lesions of the ventral hippocampus, but not the dorsal hippocampus, impair conditioned fear expression and inhibitory avoidance on the elevated T-maze. Neurobiol Learn Mem $\mathbf{8 1}$ 172-184.

Vouimba RM, Yaniv D, Richter-Levin G. 2007. Glucocorticoid receptors and $\beta$-adrenoceptors in basolateral amygdala modulate synaptic plasticity in hippocampal dentate gyrus, but not in area CA1. Neuropharmacology 52: 244-252.

Yoon T, Otto T. 2007. Differential contributions of dorsal vs. ventral hippocampus to auditory fear conditioning. Neurobiol Learn Mem 87: 464-475.

Received September 18, 2009; accepted in revised form December 3, 2009. 




\section{Role of amygdala and hippocampus in the neural circuit subserving conditioned defeat in Syrian hamsters}

Chris M. Markham, Stacie L. Taylor and Kim L. Huhman

Learn. Mem. 2010, 17:

Access the most recent version at doi:10.1101//m.1633710

References This article cites 35 articles, 9 of which can be accessed free at:

http://learnmem.cshlp.org/content/17/2/109.full.html\#ref-list-1

License

Email Alerting Receive free email alerts when new articles cite this article - sign up in the box at the Service top right corner of the article or click here. 